\title{
Article \\ Concurrent Validation of 3D Joint Angles during Gymnastics Techniques Using Inertial Measurement Units
}

\author{
Joana Barreto $^{1}\left(\mathbb{D}\right.$, César Peixoto ${ }^{2}$, Sílvia Cabral ${ }^{3}\left(\mathbb{D}\right.$, Andrew Mark Williams ${ }^{4}$, Filipe Casanova ${ }^{5,6}(\mathbb{D}$, \\ Bruno Pedro ${ }^{3}$ and António P. Veloso ${ }^{3, *}$
}

check for updates

Citation: Barreto, J.; Peixoto, C.; Cabral, S.; Williams, A.M.; Casanova, F.; Pedro, B.; Veloso, A.P. Concurrent Validation of 3D Joint Angles during Gymnastics Techniques Using Inertial Measurement Units. Electronics 2021, 10, 1251. https://doi.org/10.3390/ electronics10111251

Academic Editors: Fernanda Irrera, Giovanni Saggio, Vito Errico and Ivan Mazzetta

Received: 8 April 2021

Accepted: 17 May 2021

Published: 24 May 2021

Publisher's Note: MDPI stays neutral with regard to jurisdictional claims in published maps and institutional affiliations.

Copyright: (c) 2021 by the authors. Licensee MDPI, Basel, Switzerland. This article is an open access article distributed under the terms and conditions of the Creative Commons Attribution (CC BY) license (https:// creativecommons.org/licenses/by/ $4.0 /)$.
1 Laboratório de Perícia no Desporto, Faculdade de Motricidade Humana, CIPER, Universidade de Lisboa, 1495-751 Cruz Quebrada Dafundo, Portugal; joana.cpbarreto@gmail.com

2 Laboratório de Perícia no Desporto, Faculdade de Motricidade Humana, Universidade de Lisboa, 1495-751 Cruz Quebrada Dafundo, Portugal; cpeixoto@fmh.ulisboa.pt

3 Laboratório de Biomecânica e Morfologia Funcional, Faculdade de Motricidade Humana, CIPER, Universidade de Lisboa, 1495-751 Cruz Quebrada Dafundo, Portugal; scabral@fmh.ulisboa.pt (S.C.); bmpedro@fmh.ulisboa.pt (B.P.)

4 Department of Health and Kinesiology, College of Health, University of Utah, Salt Lake City, UT 84112, USA; mark.williams@health.utah.edu

5 Center of Research, Education, Innovation and Intervention in Sport (CIFI2D), Faculty of Sport, University of Porto, 4200-450 Porto, Portugal; fcasanova@fade.up.pt or p5661@mso365.ulp.pt

6 Faculty of Physical Education and Sport, University Lusófona, 1749-024 Lisboa, Portugal

* Correspondence: apveloso@fmh.ulisboa.pt

\begin{abstract}
There are advantages in using inertial measurement unit systems (IMUS) for biomechanical analysis when compared to $2 \mathrm{D} / 3 \mathrm{D}$ video-based analysis. The main advantage is the ability to analyze movement in the natural performance environment, preserving the ecological validity of the task. Coaches can access accurate and detailed data in real time and use it to optimize feedback and performance. Efforts are needed to validate the accuracy of IMUS. We assess the accuracy of the IMUS Xsens MVN Link system using an optoelectronic system (OS) as a reference when measuring 3D joint angles during the gymnastics round-off back handspring technique. We collected movement kinematics from 10 participants. The coefficient of multiple correlation (CMC) results showed very good and excellent values for the majority of the joint angles, except for neck flexion/extension (F/E). Root mean square errors (RMSE) were below $/$ near $10^{\circ}$, with slightly higher values for shoulder $\left(12.571^{\circ}\right)$, ankle $\left(11.068^{\circ}\right)$, thorax-thigh $\mathrm{F} / \mathrm{E}\left(21.416^{\circ}\right)$, and thorax-thigh internal/external rotation (I/E) $\left(16.312^{\circ}\right)$. Significant SPM-1D $\{\mathrm{t}\}$ differences for thorax-thigh abduction/adduction (A/A), neck, thorax-thigh, knee, shoulder and ankle F/E were demonstrated during small temporal periods. Our findings suggest that the Xsens MVN Link system provides valid data that can be used to provide feedback in training.
\end{abstract}

Keywords: biomechanics; Xsens; 3D joint kinematics; inertial measurement units; inertial sensors

\section{Introduction}

Gymnastics is an ancient sport in which movement perfection is desirable. The roundoff back handspring is a fundamental technique performed on various apparatuses (e.g., tumbling, floor, balance beam) across diverse gymnastics disciplines (e.g., Women's Artistic Gymnastics, Men's Artistic Gymnastics, TeamGym, Acrobatics and Trampoline). This technique is learned at an early age and performed at all competitive levels. Technical feedback is essential during training to improve performance and it is typically provided using $2 \mathrm{D}$ video-analysis procedures or qualitative feedback. The use of $2 \mathrm{D}$ analysis has limitations when applied to gymnastics where body rotations occur in all motion planes. However, more objective and detailed feedback requires gymnasts to be evaluated in a laboratory using Optoelectronic Systems (OS). The challenge is to examine performance using 
a practical and accurate methodology that provides precise and objective biomechanical feedback in real time and in the actual performance setting.

The OS is the most referenced approach for 3D biomechanical analysis in the laboratory [1,2]. Nevertheless, its use in an artificial, non-representative context (i.e., laboratory versus training hall), can negatively influence the true motion pattern of the athlete [3], compromising ecological validity. Moreover, it can lead to problems, such as the occlusion and loss of data from some reflective markers $[2,4,5]$ due to the body positions and rotations and because of the small volume allotted for data capture.

The use of IMUS offers a practical and cost-effective solution with the possibility of having a larger capture volume and requiring less time to prepare the participants and analyze the data, enabling data to be collected in realistic settings $[3,6]$. However, as with other measuring systems, IMUS are affected by errors associated with technology and biomechanical models [7], and accuracy varies with movement characteristics, such as angular velocity $[8,9]$.

The lack of studies using IMUS to analyze dynamic, high-velocity sports movements creates uncertainty about its validity. Without proper validation and understanding of IMUS performance, misinterpretation of the data could lead to erroneous feedback. On the other hand, knowing the task-specific degree of accuracy of IMUS may permit its extended use as a tool to optimize performance inside the gymnasium with precise data. To our current knowledge, IMUS validity for gymnastics techniques has not been previously analyzed. The purpose of this study is to assess the concurrent validity of Xsens MVN Link system (Xsens MVN, Enschede, the Netherlands) in measuring whole-body 3D joint angles, using an OS (Qualisys AB, Sweden) as the criterion during the performance of the gymnastics round-off back handspring technique. Errors between systems can emerge from differences in technologies (optical tracking versus inertial sensors) and in biomechanical models. With the literature reporting a low technological error [7], and considering our methodology in relation to the biomechanical model for OS and the improvements on sensor fusion algorithms, we expect to validate the IMUS Xsens MVN Link [10].

\section{Materials and Methods}

The sample size was determined based on previous studies $[2,11,12]$. Statistical parameters to define the sample size were an effect size of 0.50 , an alpha of 0.05 and power of 0.80 . The time spent with each gymnast at the laboratory (two hours) was also considered. Ten $(\mathrm{N}=10)$ national level gymnasts (9 female, 1 male, age $23.9 \pm 3.9$ years, height $1.57 \pm 0.37 \mathrm{~m}$; mass $53.72 \pm 4.02 \mathrm{~kg}$ ) signed their informed consent to participate in this study. The inclusion criteria were that participants (a) were able to autonomously perform the task; (b) had no restrictions due to injuries; and (c) were affiliated with a club and competed regularly. The study was conducted in accordance with the Declaration of Helsinki and was approved by the Faculty Ethics Committee (number 6/2018; 4 April 2018).

The data were collected in the laboratory using a carpet that was $12 \mathrm{~m}$ in length, $2 \mathrm{~m}$ in width and $3.5 \mathrm{~cm}$ thick. Participants wore a sporting bra (females) and tight shorts (females and males). An OS with fifteen cameras (Oqus 3+ and 7, Qualisys AB, Göteborg, Sweden) was used concurrently with an IMUS (Xsens MVN Link, Enschede, the Netherlands). The IMUS Xsens MVN Link consists of five MTx sensors (placed on the pelvis, sternum, hands and head) and twelve MTx-STR sensors (placed on the legs and upper body). Both inertial unit types contain $3 \mathrm{D}$ linear accelerometers to measure accelerations, including gravitational acceleration, 3D rate gyroscopes to measure angular velocities, 3D magnetometers to measure earth magnetic field, and a barometer to measure atmospheric pressure. The volume of capture was calibrated with a wand over a two-minute period and was accepted when the error was $\leq 1.5 \mathrm{~mm}$.

Both systems captured kinematic data simultaneously at a frequency of $240 \mathrm{~Hz}$, were time-synchronized with the Xsens Sync Station and started with a signal coming from a trigger button (pulse polarity: $5 \mathrm{~V}$ ). 
Seventeen inertial sensors were placed according to the manufacturer's recommendations directly on the skin, back of the head and sternum, shoulders (middle of the scapula spine), upper arms (lateral side above elbow), forearms (medial side of wrist), hands (posterior side), pelvis (middle of both the posterior superior iliac spines), thighs (lateral side above the knees), shanks (medial-anterior surface of the tibias), and feet (top middle) (Figure 1). The inertial sensors and wires were reinforced with adhesive. To scale the Xsens biomechanical model, anthropometric measures were collected from each participant [4].

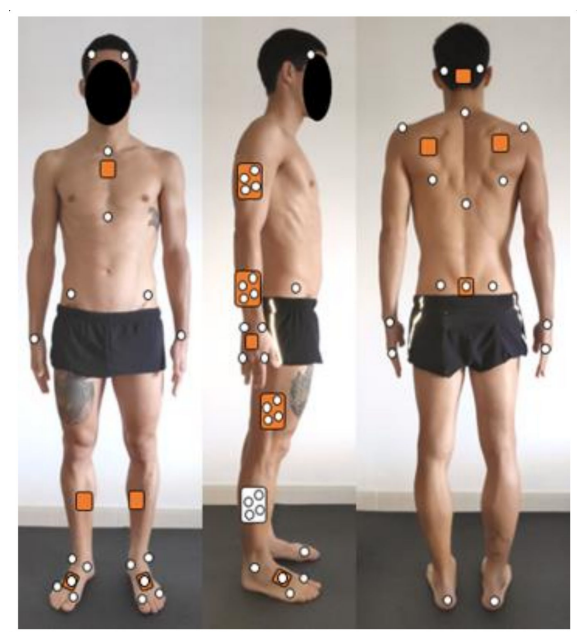

Figure 1. Placement of inertial sensors (orange squares), anatomical tracking markers (white circles) and rigid, lightweight plates (with four non-collinear reflective markers each). Rigid, lightweight plates were placed on top of inertial sensors (orange squares with white spheres), except for shanks (white squares with white spheres inside).

Anatomical markers were placed on the pelvis (anterior and posterior superior iliac spines, sacrum flat zone), thorax (jugular notch, xiphisternal joint, 7th cervical and 10th thoracic vertebras, scapula acromial edges, scapula inferior angles), head (back and front right and left aspects), feet (calcaneous posterior surface, lateral and medial malleolus, distal head of the 1st and 5th metatarsus and proximal head of the 2nd metatarsus) and hands (ulna and radius styloid processes, head of 2nd and 5th metacarpus), using doublesided tape and strengthened with adhesive (Figure 1). Rigid, lightweight plates with four markers each [13] were attached over the IMUS on the lateral side of the upper arms, forearms and thighs. In relation to the shanks (lower legs), where the IMUS were placed medially (according to the manufacturer), the rigid, lightweight plates were placed laterally to avoid marker occlusion [14] (Figure 1).

Participants performed a fifteen-minute warm-up and some practice trials to become accustomed to the equipment. The IMUS was calibrated performing N-pose + walk $+\mathrm{N}$-pose (recommended by the manufacturer) to construct the Xsens biomechanical model and define the forward pointing $X$-axis and segments orientation. The final $\mathrm{N}$-pose was also used to build the OS biomechanical model following the definitions of the Xsens model, such as the same origins, dimensions and anatomical axes orientations (Appendix A Table A1), so we can compare equivalent biomechanical models. This method was used to reduce the error that is attributed to the different biomechanical models [7]. However, the error is not completely removed since this procedure does not include corrections made by the IMUS during the walking phase of calibration.

Participants performed five trials of round-off back handsprings (Appendix A Tables A2 and A3). The trials had a mean duration of $1.53 \pm 0.09 \mathrm{~s}$. The best three trials of each participant were considered for analysis, with a total of 30 trials analyzed.

This task was chosen to capture and analyze (a) rotations in sagittal, frontal and transverse planes; (b) impact phases; and (c) aerial phases of a complex gymnastics technique (Appendix A Tables A2 and A3). Xsens data were reprocessed in HD quality in MVN 
Analyse software (Version 2019.2, Xsens, Enschede, Netherlands) and the events of interest were defined through acceleration peaks [15], representing the impacts of both hands and the dominant-side foot. Xsens MVN data were exported in MVNX format and were imported in Visual 3D (Version 6, C-Motion, Inc., Germantown, MD, USA).

Reflective marker trajectories during N-pose and round-off back handspring trials were tracked, identified and exported to c3d format, using Qualisys Track Manager (Version 2019.1, QTM, Göteborg, Sweden). Joint angles for the dominant side were calculated in the joint coordinate system [16], following a Cardan X-Y-Z (medio-lateral, antero-posterior, longitudinal) rotation sequence, using the software Visual 3D (Version 6, C-Motion, Inc., Germantown, MD, USA). Joint angles were filtered with a 4th order low pass Butterworth filter in Visual 3D (Version 6, C-Motion, Inc., Germantown, MD, USA). The cut-off frequency was calculated using the Fast Fourier Transform Technique (residual analysis) (Version 4.4.2, Acqknowledge software) and the data were filtered with a cut-off frequency of $14 \mathrm{~Hz}$ (neck and arms) and $21 \mathrm{~Hz}$ (thorax and legs).

Neck, shoulder and thorax-thigh abduction/adduction (A/A), flexion/extension $(\mathrm{F} / \mathrm{E})$, internal/external rotation (I/E) and elbow, wrist, knee and ankle F/E were considered for analysis due to the importance of these joints movements in performing this technique. A particular case respecting the biomechanical model definitions is the pelvis. Since joint angles between pelvis-thigh and pelvis-thorax are not relevant for the task analyzed, the thorax-thigh joint angles were calculated as the angle between the thorax and thigh. This angle defines the body position of the gymnast and it is a pertinent factor for movement analysis and, consequently, for scoring during a competition. All data were time normalized to the period between the contact of the leading foot with the floor carpet in the beginning of the round-off and the contact of feet with the floor carpet at the end of the back handspring.

With the aim to assess the concurrent validity of the IMUS, the coefficient of multiple correlation (CMC mean $\pm \mathrm{SD}$ ) and the root mean square errors (RMSE mean $\pm \mathrm{SD}$ ) were calculated. Joint angle data obtained with OS and IMUS were time normalized. The average curves for each joint plane of each participant were calculated for IMUS and OS. Then, the RMSE and the CMC were calculated between each mean curve of IMUS and OS. CMC reports measures of wave similarity between the OS and IMUS. RMSE \pm SD is a combination of accuracy and precision between the two systems. We consider that a $\mathrm{CMC} \geq 0.80$ and $\mathrm{aMSE} \leq 10^{\circ}$ [8] are acceptable since the Code of Points [17] attributes a minor execution deduction for differences of up to $15^{\circ}$ from the body shape definitions. A two-tailed paired sample t-test was computed for every time point, based on the SPM-1D Random Field Theory [18,19]. SPM-1D $\{t\}$ allowed to assess whether statistically significant differences occur along the 1-dimension waveforms of both systems. Despite the fact that the majority of validation studies reported CMC values due to its utility [20], it is recommended to evaluate the validity of IMUS by measuring the continuous time-series parameters [14], using, for example, SPM-1D software.

\section{Results}

In total, 30 trials were considered for analysis. An example of a trial of one participant is presented in Figure 2 and an example of three trials for one subject is present in Figure A1.

The results indicate a high RMSE for joint planes shoulder F/E, thorax-thigh F/E and ankle F/E (Table 1), together with significant differences, according to our criteria, between the IMUS and OS (Figure 3a,b). SPM-1D $\{t\}$ also demonstrates significant differences for joint planes thorax-thigh A/A, neck F/E and knee F/E (Figure 3a,b), despite the very good results for $\mathrm{CMC}$ and a RMSE $<10^{\circ}$. A poor $\mathrm{CMC}$ was obtained for joint plane neck A/A (Table 1), revealing different waveforms of the two systems data. The remaining joint planes (i.e., shoulder A/A, shoulder and thorax-thigh I/E, elbow, wrist and neck F/E) has acceptable CMC and RMSE (Table 1) and no significant differences between IMUS and OS, according to our criteria (Figure $3 a, b)$. 

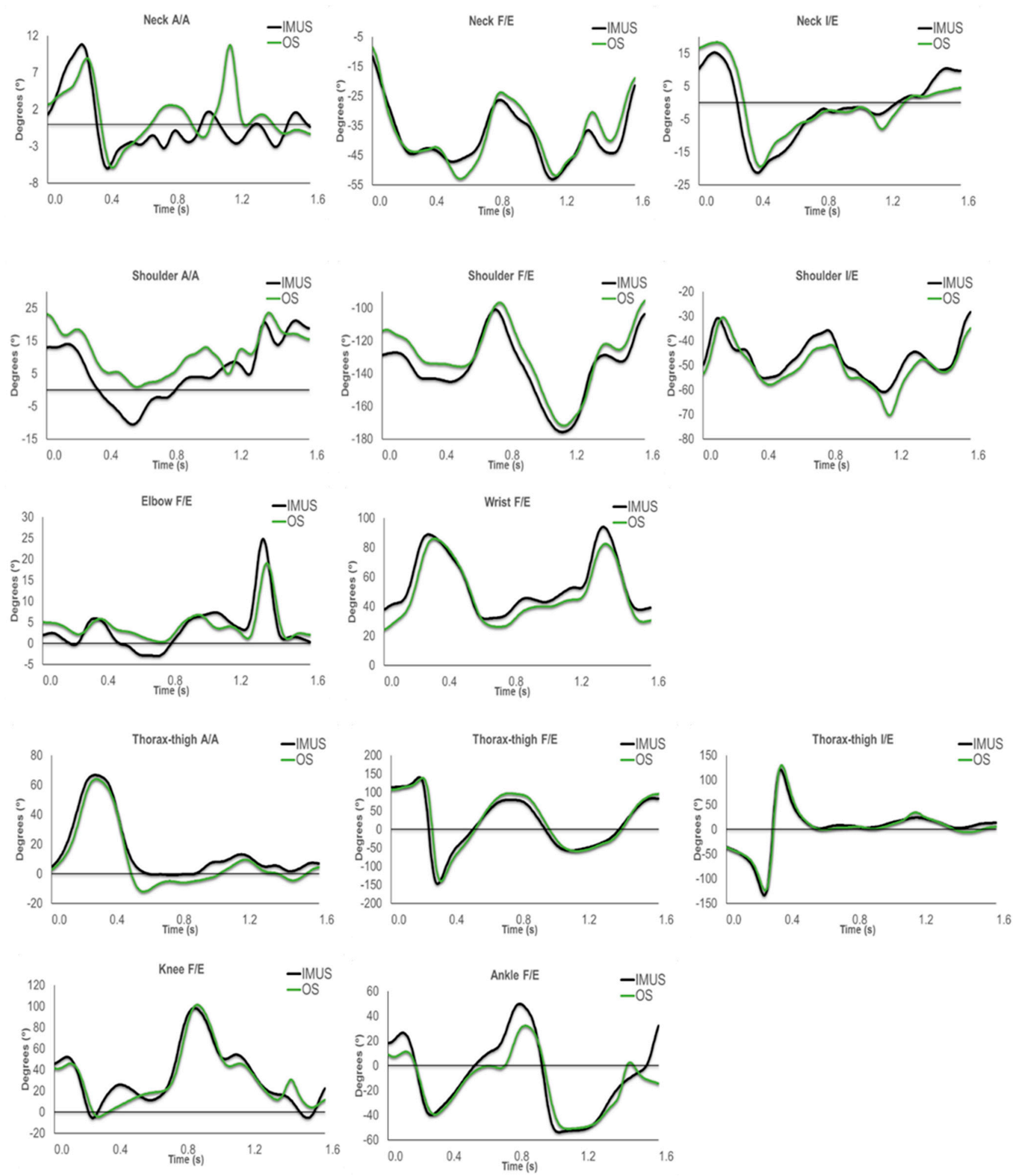

Figure 2. Joint angles curves from IMUS and OS for a trial of one participant performing the round-off back handspring. Note: $\mathrm{A} / \mathrm{A}=$ abduction/adduction; $\mathrm{F} / \mathrm{E}=$ flexion/extension; $\mathrm{I} / \mathrm{E}=$ internal/external rotation. 
Table 1. Coefficient of multiple correlation, root mean square error and standard deviation values between Xsens MVN and Qualisys.

\begin{tabular}{ccccc}
\hline Joint and Plane & CMC & CMC Std Dev. & RMSE $\left(^{\circ}\right.$ ) & RMSE Std Dev. \\
\hline Neck A/A & $0.54^{*}$ & 0.14 & 5.79 & 2.89 \\
Neck F/E & 0.98 & 0.02 & 7.21 & 3.70 \\
Neck I/E & 0.85 & 0.18 & 7.48 & 4.00 \\
Shoulder A/A & 0.88 & 0.12 & 9.86 & 3.90 \\
Shoulder F/E & 0.99 & 0.00 & $12.57^{*}$ & 3.42 \\
Shoulder I/E & 0.98 & 0.01 & 8.46 & 4.59 \\
Elbow F/E & 0.96 & 0.04 & 4.20 & 1.38 \\
Wrist F/E & 0.99 & 0.00 & 6.91 & 2.00 \\
Thorax-thigh A/A & 0.96 & 0.02 & 8.19 & 2.37 \\
Thorax-thigh F/E & 0.97 & 0.03 & $21.42 *$ & 10.31 \\
Thorax-thigh I/E & 0.90 & 0.06 & $16.31^{*}$ & 4.62 \\
Knee F/E & 0.98 & 0.01 & 8.36 & 3.42 \\
Ankle F/E & 0.95 & 0.02 & $11.07^{*}$ & 1.82 \\
\hline
\end{tabular}

Note: CMC = coefficient of multiple correlation; Std Dev. = standard deviation; RMSE = root mean square error; $\mathrm{A} / \mathrm{A}=$ abduction/adduction; $\mathrm{F} / \mathrm{E}=$ flexion/extension; $\mathrm{I} / \mathrm{E}=$ internal/external rotation. ${ }^{*}$ denotes poor $\mathrm{CMC}$ and RMSE above our criteria value of $10^{\circ}$.
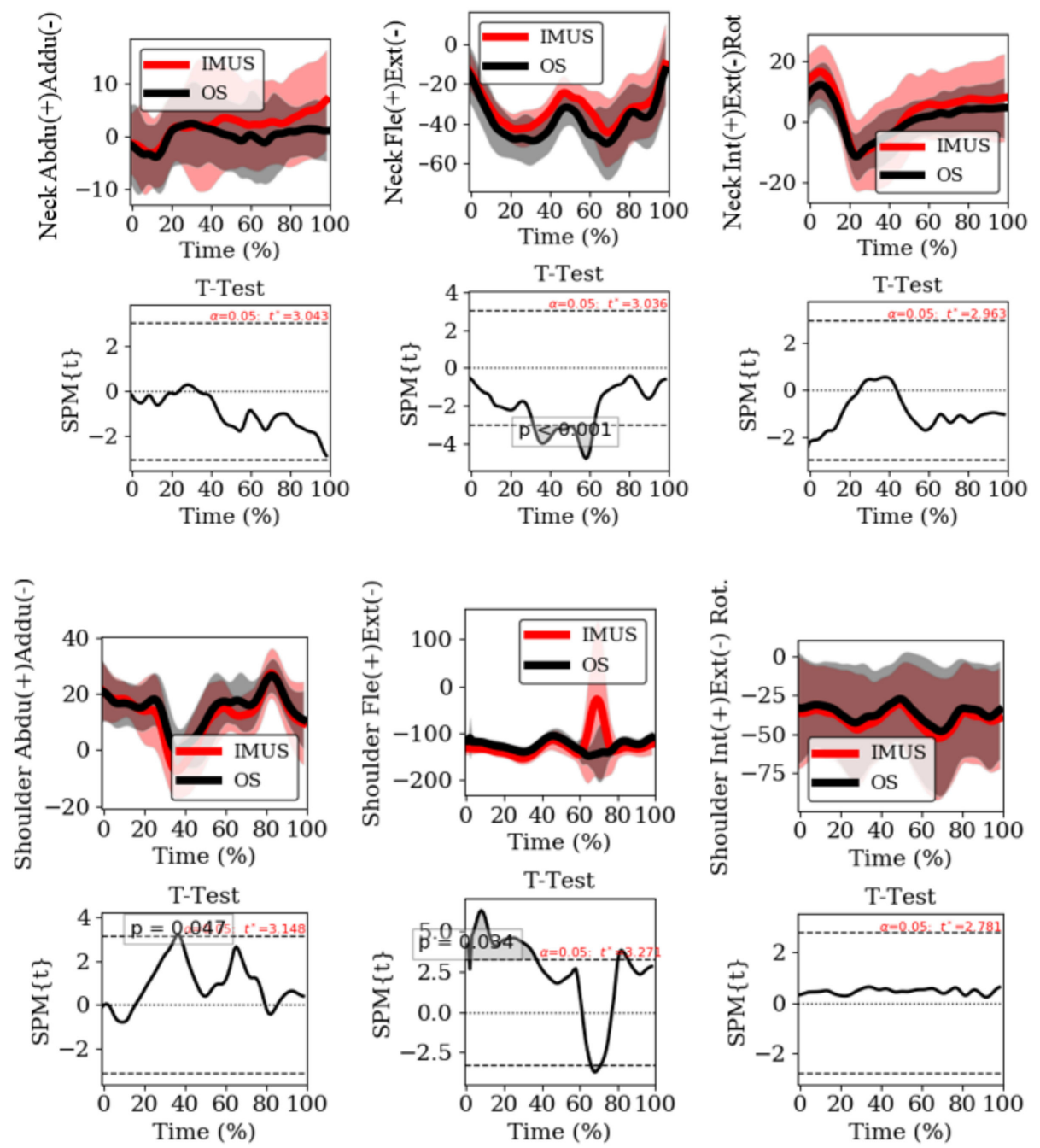

Figure 3. Conts. 

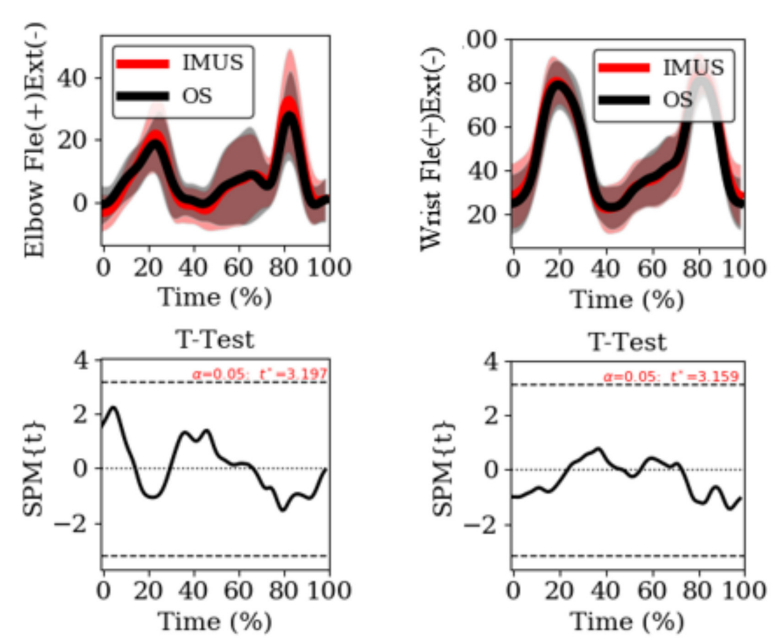

(a)
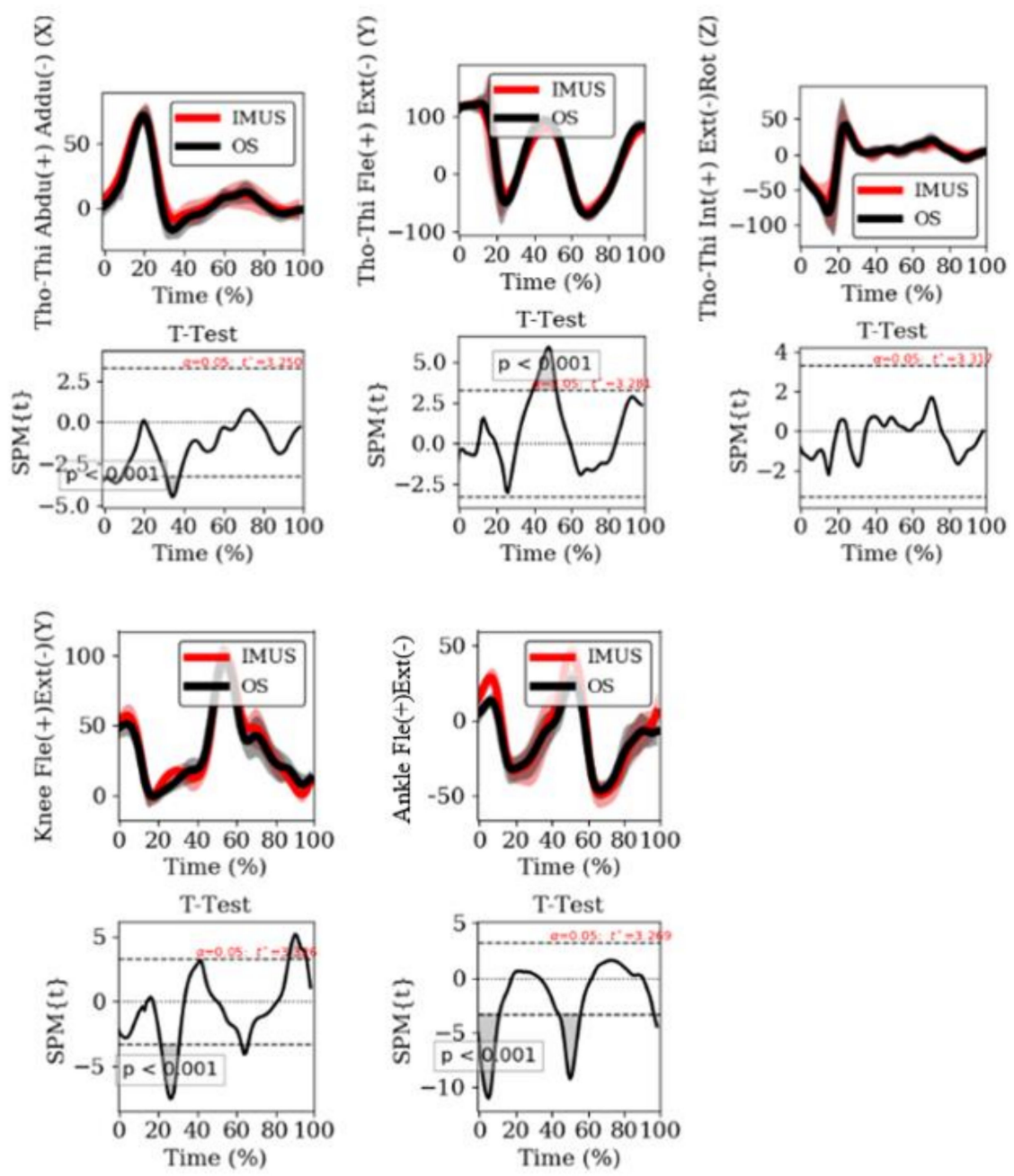

(b)

Figure 3. (a) Mean joint kinematic angles time series with SD clouds for upper body. (b) Mean joint kinematic angles time series with SD clouds for thorax and lower body. SPM-1D $\{t\}$ is the mean difference curve normalized by the sample size normalized variance. The dotted horizontal lines represent the Random Field Theory threshold for significance. $p$-values indicate the likelihood that a random process of the same temporal smoothness would be produce a supra-threshold cluster of the observed size. Note: Tho-Thi $=$ Thorax-thigh, $\mathrm{Abdu}(+) / \operatorname{Addu}(-)=\operatorname{Abduction}(+) / \operatorname{Adduction}(-)$, Fle $(+) / \operatorname{Ext}(-)=$ Flexion(+)/Extension( $(-), \operatorname{Int}(+) / \operatorname{Ext}(-)$ Rot. $=$ Internal(+)/External( -$)$ Rotation. 


\section{Discussion and Conclusions}

We assessed the criterion-related validity of an IMUS (Xsens MVN Link) in measuring joint angles, using an OS as reference, as gymnasts performed the round-off back handspring technique.

This study presents limitations, such as the non-ecological environment where the task round-off back handspring was performed (i.e., laboratory), which can affect the motor patterns of the participants.

Comparing our results (Table 1), a lower RMSE was demonstrated for shoulder F/E during military tasks [21] and manual material handling tasks [7], as well as for thoraxthigh F/E during walking [22,23], running [22], postural disturbances in a treadmill [11] and during a repetitive movement test [24]. In addition, lower results for RMSE were found for ankle F/E during walking [22], military tasks [21] and squat and counter movement jumps [25], together with thorax-thigh I/E. The high RMSE for these joint planes are reinforced by the significant differences found on SPM-1D $\{t\}$ (except for thorax-thigh I/E), which are in accordance with the results obtained during the tennis forehand drive [26], although the authors reported lower RMSE. These results can be explained by two factors. First, although we built the OS biomechanical model with the same segment definitions as the IMUS biomechanical model (Appendix A Table A1), this type of error was reduced but not completely removed, contrary to studies that isolated technological error by completely eliminating the error from the two different biomechanical models [7,21]. Second, the high angular velocities of the movement may have contributed to our results. However, we obtained a lower RMSE for shoulder F/E than a study that compared two different biomechanical models during a repetitive movement test [24].

Despite the acceptable RMSE and no significant differences between IMUS and OS for neck A/A, the waveform similarity is poor (Table 1), contrasting to an excellent correlation found during active movements of the head in a seated position [24]. We believe that the existent differences between the two biomechanical models contributed to this result, leading to the conclusion that, for neck A/A, both systems do not agree in terms of movement pattern. However, the reduced number of studies evaluating IMUS performance for the neck joint [27] limits the comparison with our results.

Although the remaining joint planes present acceptable RMSE and good to excellent $\mathrm{CMC}$ (i.e., $\leq 10^{\circ}$ and $\geq 0.80$, respectively), joint planes knee $\mathrm{F} / \mathrm{E}$, neck $\mathrm{F} / \mathrm{E}$ and thorax-thigh A/A have significant differences (Figure 3a,b) for very short time intervals, despite the very good CMC and acceptable RMSE. Significant differences between both systems were also reported for knee F/E in the study of the tennis forehand drive (26). These differences are associated with the RMSE that, even though is acceptable, it is close to the criteria value (i.e., $\leq 10^{\circ}$ ) (Table 1$)$.

Lower RMSE were observed for shoulder A/A during military tasks [21], the tennis forehand drive [26], and manual material handling tasks [7]. The latter paper also reported lower RMSE for elbow and wrist F/E. The CMC for elbow F/E during swimming [12], manual material handling tasks [7] and military tasks [21] are in accordance to our results. As we reported, no significant differences were found for elbow and wrist F/E [26]. Similar RMSE for shoulder A/A and knee F/E were observed in studies that reported the total error (i.e., errors from different technologies and different biomechanical models), on a repetitive movement task [24], and during gait [22,28]. We consider that the high angular velocities that characterize these joints during the round-off back handspring may have increased the errors between the two systems when compared with the majority of the studies, although they present acceptable CMC $\geq 0.88$ and RMSE $\leq 10^{\circ}$ for our purposes. Surprisingly, a study that isolated technological error (i.e., eliminated the error that emerges from the different biomechanical models), reported a high RMSE for knee F/E during military tasks [21], which may be attributed to the different method that we used to reduce the error from the two different biomechanical models. Finally, similar to our study, very good and excellent $\mathrm{CMC}$ were shown during walking, stair ascent and descent [29] for hip $\mathrm{A} / \mathrm{A}$ and I/E, knee F/E and ankle F/E. 
In the present study, we conclude that the Xsens MVN Link system has good performance in measuring $3 \mathrm{D}$ joint angles during the round-off back handspring, except for thorax-thigh $\mathrm{F} / \mathrm{E}$, translating into a very high error compared to the OS. Although RMSE for shoulder F/E, thorax-thigh I/E and ankle F/E are above the value that we defined as acceptable for our purpose, we believe that the IMUS can be used in training since the values are closer to the criteria value from Code of Points for minor deductions $\left(15^{\circ}\right)$. In relation to neck A/A, despite the acceptable error between the two systems, we advise not to use Xsens MVN Link to analyze the pattern of movement since the similarity between the waveforms (CMC) of OS and IMUS is poor.

Additionally, our study reports a combination of errors from technology and biomechanical models, since we did not completely remove the error associated with the biomechanical models. We believe that the differences between the two biomechanical models affect joints differently, with joints with more amplitude of movement (e.g., shoulder, thorax-thigh, knee and ankle F/E) being the most affected and, consequently, registering a higher RMSE. Although the method used to align the biomechanical models was not the most effective (i.e., compared to the method of angular velocities [30,31]), it was still possible to demonstrate that Xsens MVN Link is a suitable instrument to measure movement kinematics in sports. Our findings are a significant step toward the use of this technology in a practical context. Beyond the advantages of IMUS, such as the small amount of time needed to prepare the participant and to calibrate the system, the system allows data to be provided in real time in practice. However, it would be beneficial for the gymnastics-specific context if the system could provide automatic classification of the performance (e.g., classification of the body position according to joint angles), giving direct feedback to the coach and gymnast.

We recommend the application of Xsens MVN Link to provide participants with technical feedback in training contexts of acrobatic sports (e.g., gymnastics, figure skating, parkour, and jump rope) but not to compare performances between participants nor to provide extremely detailed technical feedback.

We conclude that, according to our methodology, the IMUS are not suitable to measure thorax-thigh $\mathrm{F} / \mathrm{E}$ and caution should be taken when considering data from shoulder $\mathrm{F} / \mathrm{E}$, thorax-thigh I/E and ankle F/E. Future studies using more effective methods to eliminate the error from biomechanical models [30,31], especially for the thorax-thigh joint, should further elevate confidence in using Xsens MVN Link to assess and compare gymnasts.

Author Contributions: Methodology, J.B.; S.C. and B.P.; software, A.P.V.; formal analysis, J.B.; S.C. and B.P.; investigation, J.B.; writing-original draft preparation, J.B.; writing-review and editing, S.C.; C.P.; B.P.; A.M.W.; F.C. and A.P.V.; supervision, A.P.V. and C.P. All authors have read and agreed to the published version of the manuscript.

Funding: Grant UIDB/00447/2020 to CIPER—Centro Interdisciplinar para o Estudo da Performance Humana (unit 447).

Institutional Review Board Statement: The study was conducted according to the guidelines of the Declaration of Helsinki, and approved by the Institutional Ethics Committee of Faculty of Human Kinetics-University of Lisbon (approval number 6/2018; 4 April 2018).

Informed Consent Statement: Informed consent was obtained from all subjects involved in the study.

Data Availability Statement: The data presented in this study are available on request from the corresponding author.

Conflicts of Interest: The authors declare no conflict of interest. 


\section{Appendix A}

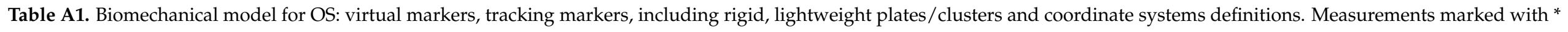
refer to the anthropometric measurements taken from the participant during the session. Measurements marked with ** are estimated through regression equations [4].

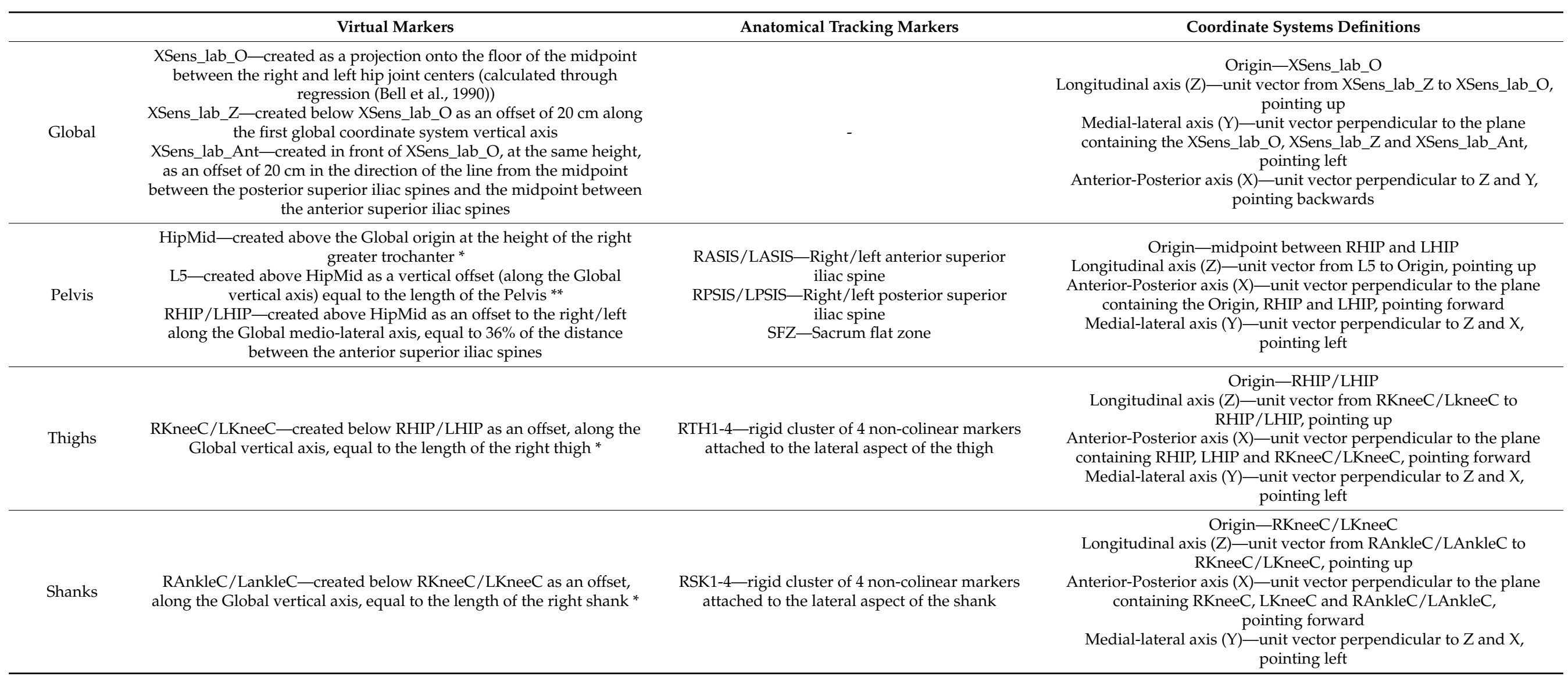


Table A1. Conts.

\section{Virtual Markers}

RdistalFoot/LdistalFoot—created in front of RAnkleC/LAnkleC as an offset, along the Global anterior axis, equal to the length of

RToebase/LToebase-created bellow RdistalFoot/LdistalFoot as an offset of $5 \mathrm{~cm}$, along the Global vertical axis

Head_Mid—created above C7 as an offset, along the Global vertical axis, equal to half the head's length **

C7-created above L5 as an offset, along the Global vertical axis, equal to the length of trunk

Thorax

Posterior-created as an offset of $10 \mathrm{~cm}$ from the Global origin, along the Global posterior axis and at the height of the right greater trochanter *

RClavOrigin/LClavOrigin—created as an upward and lateral offset from L5, respectively along the Global vertical and medial-lateral axis, to the origin of the clavicle **

RShoulderC/LShoulderC—created as an offset to the right/left of

RClavOrigin, along the Global medial-lateral axis, equal to the length the right clavicle*

RElbowC/LElbowC—created below RShoulderC/LShoulderC as an offset, along the Global vertical axis, equal to the length of the right upper arm *

RFA1-4-rigid cluster of 4 non-colinear markers attached to the lateral aspect of the forearm
R_FM1/L_FM1_distal head of the 1st metatarsus R_FM2/L_FM2-proximal head of the 2nd metatarsus

R_FM5/L_FM5-distal head of the 5th metatarsus R_FAL/L_FAL_apex of the lateral malleolus R TAM/L_TAM-apex of the medial malleolus

R FCC/L FCC - posterior surface of the calcaneus

R_F_head/L_F_head—right/left aspect of the forehead

R_B_head/L_B_head-right/left aspect of the back of the head

\section{AXIF-xiphisternal joint}

CLAV-jugular notch

C7-spinous process of 7th cervical vertebra

10 - spinous process of the 10th thoracic vertebra

R_SIA/L_SIA-inferior angle of the scapula

R_SAE/L_SAE_-scapula acromial edge

RUA1-4-rigid cluster of 4 non-colinear markers

attached to the lateral aspect of the upper arm
RHandTip/LHandTip—created below RWristC/LWristC as an offset, along the Global vertical axis, equal to $40 \%$ of the length of the right hand ${ }^{* *}$
R/L_USP: Ulna styloid process R/L_RSP: radius styloid process R/L_HM2: head of 2nd metacarpus R/L_HM5: head of 5th metacarpus

\section{Coordinate Systems Definitions}

Origin-RAnkleC/LAnkleC

Anterior-Posterior axis $(\mathrm{X})$ - unit vector from RAnkleC/LAnkleC to RdistalFoot/LdistalFoot, pointing forward

Medial-lateral axis $(\mathrm{Y})$ - unit vector perpendicular to the plane containing RAnkleC/LAnkleC, RdistalFoot/LdistalFoot and RToebase/LToebase, pointing left

Longitudinal axis $(\mathrm{Z})$ - unit vector perpendicular to $\mathrm{X}$ and $\mathrm{Y}$, pointing up

\section{Origin-C7}

Longitudinal axis (Z) — unit vector from C7 to Head_Mid, pointing u Medial-lateral axis $(\mathrm{Y})$ - unit vector perpendicular to the plane containing C7, Head_Mid and Posterior, pointing left

Anterior-Posterior axis $(\mathrm{X})$ - unit vector perpendicular to $\mathrm{Y}$ and $\mathrm{Z}$, pointing forward

Longitudinal axis (Z) - unit vector from L5 to C7, pointing up Medial-lateral axis $(\mathrm{Y})$ - unit vector perpendicular to the plane containing L5, C7 and Posterior, pointing left

Anterior-Posterior axis $(\mathrm{X})$ - unit vector perpendicular to $\mathrm{Y}$ and $\mathrm{Z}$, pointing forward

Origin-RShoulderC/LShoulderC

Longitudinal axis (Z) - unit vector from RElbowC/LElbowC to RShoulderC/LShoulderC, pointing up

Anterior-Posterior axis $(\mathrm{X})$ - unit vector perpendicular to the plane Anterion-Por containing RShoulderC/LShoulderC, REI
pointing forward

Medial-lateral axis (Y) - unit vector perpendicular to $\mathrm{Z}$ and $\mathrm{X}$, pointing left

Origin—RElbowC/LElbowC

Longitudinal axis (Z) - unit vector from RWristC/LWristC to RElbowC/LElbowC, pointing up

Medial-lateral axis $(\mathrm{Y})$ - unit vector perpendicular to the plane containing RElbowC/LElbowC, RWristC/LWristC and C7, pointing backward/forward

Anterior-Posterior axis $(\mathrm{X})$ - unit vector perpendicular to $\mathrm{Z}$ and $\mathrm{X}$ pointing medially

\section{Origin-RWristC/LWristC}

Longitudinal axis (Z) - unit vector from RHandTip/LHandTip to RWristC/LWristC, pointing up

Medial-lateral axis $(\mathrm{Y})$ - unit vector perpendicular to the plane containing RHandTip/LHandTip, RWristC/LWristC and C7, pointing backward/forward

Anterior-Posterior axis $(\mathrm{X})$ - unit vector perpendicular to $\mathrm{Z}$ and $\mathrm{X}$, pointing medially 
Table A2. Description of round off: goals, phases of movement, technical description, mechanical actions, segments angles and frames of interest.

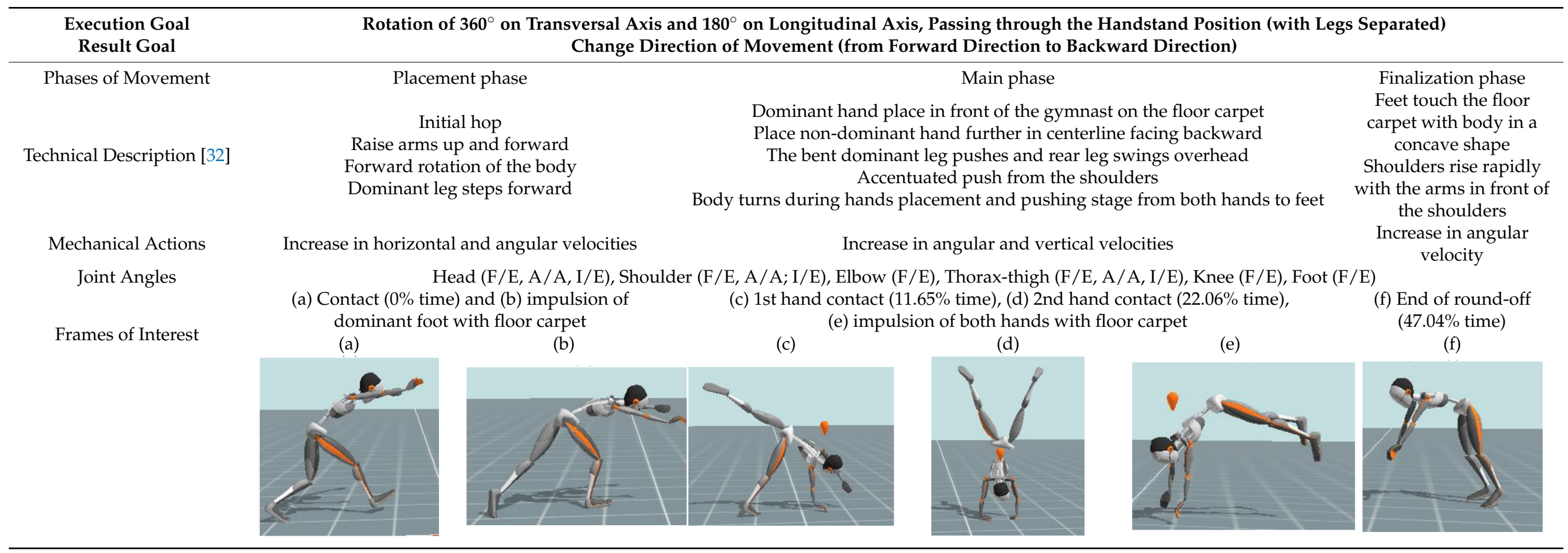


Table A3. Description of back handspring: goals, phases of movement, technical description, mechanical actions, segments angles and frames of interest.

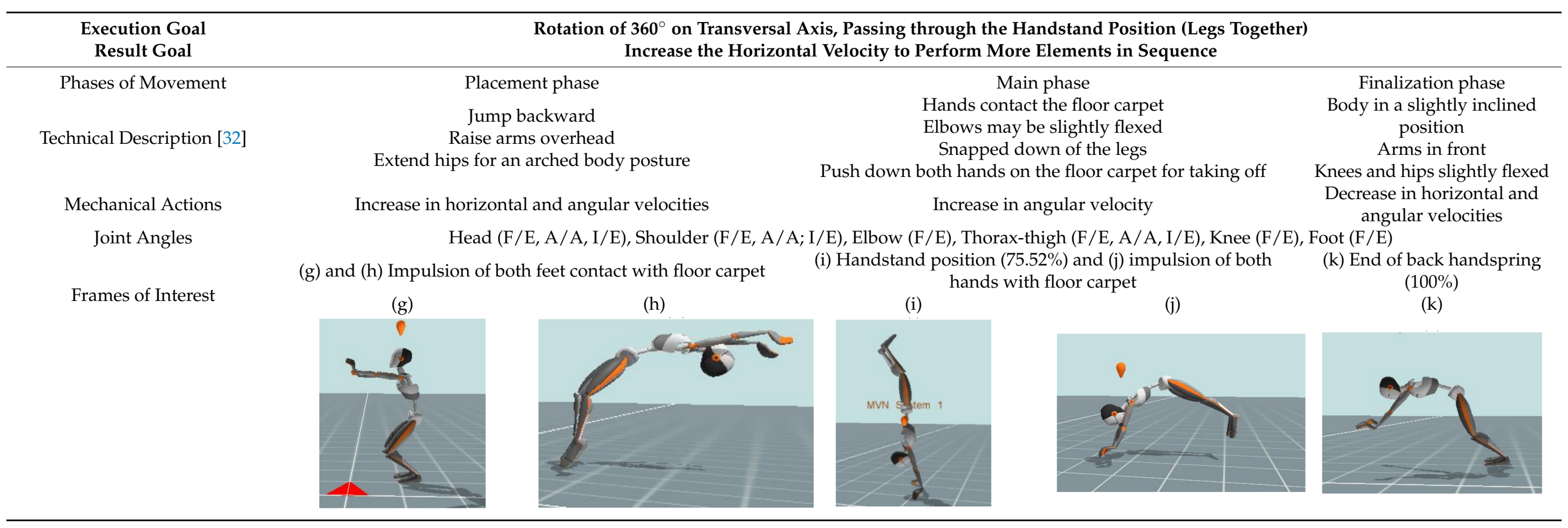



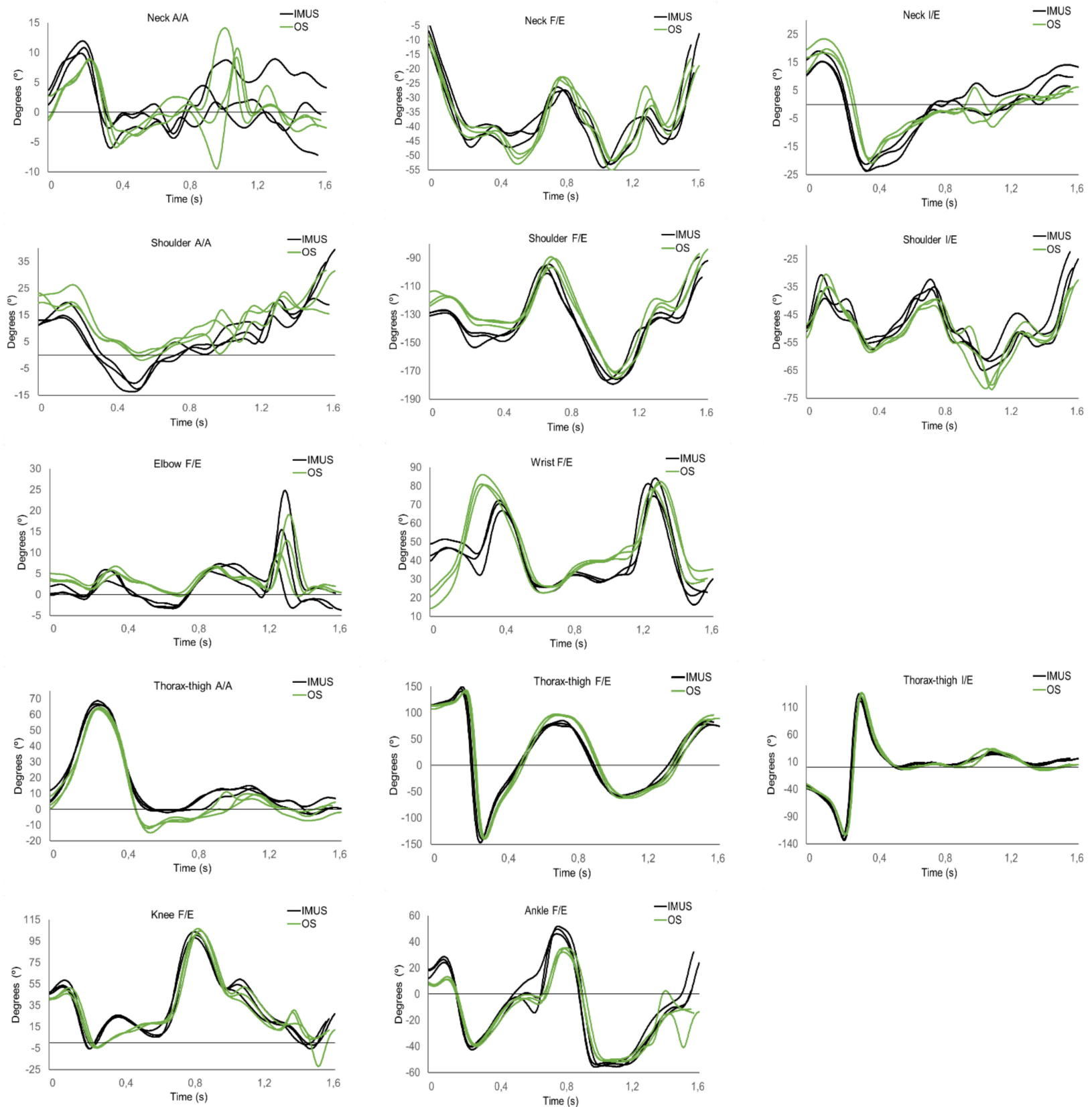

Figure A1. Joint angles measured by IMUS and OS for three trials of round-off back handsprings for one participant.

\section{References}

1. Kruk, E.; Reijne, M. Accuracy of human motion capture systems for sport applications; state-of-the-art review. Eur. J. Sport Sci. 2018, 18, 806-819. [CrossRef] [PubMed]

2. Horenstein, R.; Lewis, C.; Yan, S.; Halverstadt, A.; Shefelbine, S. Validation of magneto-inertial measuring units for measuring hip joint angles. J. Biomech. 2019, 91, 170-174. [CrossRef]

3. Koska, D.; Gaudel, J.; Hein, T.; Maiwald, C. Validation of an inertial measurement unit for the quantification of rearfoot kinematics during running. Gait Posture 2018, 64, 135-140. [CrossRef]

4. Roetenberg, D.; Luinge, H.; Slycke, P. Xsens MVN: Full 6DOF Human Motion Tracking Using Miniature Inertial Sensors. Available online: https://www.researchgate.net/profile/Per-Slycke/publication/239920367_Xsens_MVN_Full_6DOF_human_ motion_tracking_using_miniature_inertial_sensors/links/Of31752f1f60c20b18000000/Xsens-MVN-Full-6DOF-human-motiontracking-using-miniature-inertial-sensors.pdf (accessed on 6 December 2019). 
5. Chambers, R.; Gabbett, T.J.; Cole, M.H.; Beard, A. The Use of Wearable Microsensors to Quantify Sport-Specific Movements. Sport Med. 2015, 45, 1065-1081. [CrossRef]

6. Camomilla, V.; Bergamini, E.; Fantozzi, S.; Vannozzi, G. Trends supporting the in-field use of wearable inertial sensors for sport performance evaluation: A systematic review. Sensors 2018, 18, 873. [CrossRef]

7. Robert-Lachaine, X.; Mecheri, H.; Larue, C.; Plamondon, A. Validation of inertial measurement units with an optoelectronic system for whole-body motion analysis. Med. Biol. Eng. Comput. 2017, 55, 609-619. [CrossRef]

8. Lebel, K.; Boissy, P.; Nguyen, H.; Duval, C. Inertial measurement systems for segments and joints kinematics assessment: Towards an understanding of the variations in sensors accuracy. Biomed. Eng. Online 2017, 16, 1-16. [CrossRef]

9. Kim, S.; Nussbaum, M.A. Performance evaluation of a wearable inertial motion capture system for capturing physical exposures during manual material handling tasks. Ergonomics 2013, 56, 314-326. [CrossRef]

10. Schepers, M.; Giuberti, M.; Bellusci, G. Xsens MVN: Consistent Tracking of Human Motion Using Inertial Sensing; Technical Report; Xsens Technologies B.V: Enschede, The Netherlands, March 2018; pp. 1-8. Available online: https://www.researchgate. net/profile/Martin-Schepers/publication/324007368_Xsens_MVN_Consistent_Tracking_of_Human_Motion_Using_Inertial_ Sensing/links/5ab8be2f0f7e9b68ef51f7ba/Xsens-MVN-Consistent-Tracking-of-Human-Motion-Using-Inertial-Sensing.pdf (accessed on 14 May 2021).

11. Miller, E.; Kaufman, K. Cross-sectional validation of inertial measurement units for estimating trunk flexion kinematics during treadmill disturbances. Med. Eng. Phys. 2019, 70, 51-54. [CrossRef]

12. Fantozzi, S.; Giovanardi, A.; Magalhães, F.A.; Di Michele, R.; Cortesi, M.; Gatta, G. Assessment of three-dimensional joint kinematics of the upper limb during simulated swimming using wearable inertial-magnetic measurement units. J. Sports Sci. 2016, 34, 1073-1080. [CrossRef]

13. Cappozzo, A.; Cappello, A.; Croce, U.D.; Pensalfini, F. Surface-marker cluster design criteria for 3-d bone movement reconstruction. IEEE Trans. Biomed. Eng. 1997, 44, 1165-1174. [CrossRef] [PubMed]

14. Blair, S.; Duthie, G.; Robertson, S.; Hopkins, W.; Ball, K. Concurrent validation of an inertial measurement system to quantify kicking biomechanics in four football codes. J. Biomech. 2018, 73, 24-32. [CrossRef] [PubMed]

15. Mo, S.; Chow, D.H.K. Accuracy of three methods in gait event detection during overground running. Gait Posture 2018, 59, 93-98. [CrossRef] [PubMed]

16. Grood, E.S.; Suntay, W.J. A Joint Coordinate System for the Clinical Description of Thre-Dimensional Motions: Application to the Knee. J. Biomech. 1983, 105, 136-144. [CrossRef]

17. Sjostrand, P.; Lemmetty, H.; Hughes, K.; Gryga, P.; Jónsdóttir, S. 2017-2021 Code of Points Seniors and Juniors Teamgym. 2019. Available online: https:/ / gymtranet.dk/media/4720/ueg-teamgym-cop-2017-revision-a-may-2018.pdf (accessed on 14 May 2021).

18. Pataky, T. One-dimensional statistical parametric mapping in Python. Comput. Methods Biomech. Biomed. Engin. 2012, 15, $295-301$. [CrossRef]

19. Pataky, T.; Vanrenterghem, J.; Robinson, M. The probability of false positives in zero-dimensional analyses of one-dimensional kinematic, force and EMG trajectories. J. Biomech. 2016, 49, 1468-1476. [CrossRef]

20. Cuesta-Vargas, A.I.; Galán-Mercant, A.; Williams, J.M. The use of inertial sensors system for human motion analysis. Phys. Ther. Rev. 2010, 15, 462-473. [CrossRef]

21. Mavor, M.P.; Ross, G.B.; Clouthier, A.L.; Karakolis, T.; Graham, R.B. Validation of an IMU suit for military-based tasks. Sensors 2020, 20, 4280. [CrossRef]

22. Nüesch, C.; Roos, E.; Pagenstert, G.; Mündermann, A. Measuring joint kinematics of treadmill walking and running: Comparison between an inertial sensor based system and a camera-based system. J. Biomech. 2017, 57, 32-38. [CrossRef]

23. Tadano, S.; Takeda, R.; Miyagawa, H. Three dimensional gait analysis using wearable acceleration and gyro sensors based on quaternion calculations. Sensors 2013, 13, 9321-9343. [CrossRef]

24. Duc, C.; Salvia, P.; Lubansu, A.; Feipel, V.; Aminian, K. A wearable inertial system to assess the cervical spine mobility: Comparison with an optoelectronic-based motion capture evaluation. Med. Eng. Phys. 2014, 36, 49-56. [CrossRef]

25. Teufl, W.; Miezal, M.; Taetz, B.; Frohlichi, M.; Bleser, G. Validity of inertial sensor based 3D joint kinematics of static and dynamic sport and physiotherapy specific movements. PLoS ONE 2019, 14, e0213064. [CrossRef]

26. Pedro, B.; Cabral, S.; Veloso, A.P. Concurrent validity of an inertial measurement system in tennis forehand drive. J. Biomech. 2021, 121, 110410. [CrossRef]

27. Poitras, I.; Dupuis, F.; Bielmann, M.; Campeau-Lecours, A.; Mercier, C.; Bouyer, L.J.; Roy, J.S. Validity and reliability of wearable sensors for joint angle estimation: A systematic review. Sensors 2019, 19, 1555. [CrossRef]

28. Bessone, V.; Höschele, N.; Schwirtz, A.; Seiberl, W. Validation of a new inertial measurement unit system based on different dynamic movements for future in-field applications. Sport Biomech. 2019, 1-16. [CrossRef] [PubMed]

29. Zhang, J.; Novak, A.; Brouwer, B.; Li, Q. Concurrent validation of Xsens MVN measurement of lower limb joint angular kinematics. Physiol. Meas. 2013, 34, N63-N69. [CrossRef]

30. De Vries, W.; Veeger, H.; Baten, C.; van der Helm, F. Magnetic distortion in motion labs, implications for validating inertial magnetic sensors. Gait Posture 2009, 29, 535-541. [CrossRef] 
31. Mecheri, H.; Robert-Lachaine, X.; Larue, C.; Plamondon, A. Evaluation of Eight Methods for Aligning Orientation of Two Coordinate Systems. J. Biomech. Eng. 2016, 138, 2-5. [CrossRef]

32. Mack, M.; Federbusch, S.; Ferber, M.; Heinen, T. Movement prototypes and their relationship in the performance of a gymnastics floor routine. J. Hum. Sport Exerc. 2019, 1-16. [CrossRef] 